\title{
Preoperative quantification of aortic valve stenosis: comparison of 64-slice computed tomography with transesophageal and transthoracic echocardiography and size of implanted prosthesis
}

\author{
Katarzyna Mizia-Stec $\cdot$ Piotr Pysz • Marek Jasiński • Tomasz Adamczyk • \\ Agnieszka Drzewiecka-Gerber • Artur Chmiel • Michał Krejca • \\ Andrzej Bochenek • Stanisław Woś • Maciej Sosnowski • Zbigniew Gąsior • \\ Maria Trusz-Gluza $\cdot$ Michał Tendera \\ Received: 19 July 2010/Accepted: 23 December 2010/Published online: 30 January 2011 \\ (C) The Author(s) 2011. This article is published with open access at Springerlink.com
}

\begin{abstract}
Precise measurements of aortic complex diameters are essential for preoperative examinations of patients with aortic stenosis (AS) scheduled for aortic valve (AV) replacement. We aimed to prospectively compare the accuracy of transthoracic echocardiography (TTE), transoesophageal echocardiography (TEE) and multi-slice computed tomography (MSCT) measurements of the AV complex and to analyze the role of the multi-modality aortic annulus diameter (AAd) assessment in the selection
\end{abstract}

K. Mizia-Stec $(\bowtie) \cdot$ P. Pysz · T. Adamczyk ·

A. Drzewiecka-Gerber · A. Chmiel · M. Sosnowski ·

Z. Gąsior · M. Trusz-Gluza · M. Tendera

Departments of Cardiology, Medical University of Silesia,

Ziołowa 45/47, 40-635 Katowice, Poland

e-mail: kmizia@op.pl

P. Pysz

e-mail: pysze@op.pl

T. Adamczyk

e-mail: tomasz.adamczyk@gazeta.pl

A. Drzewiecka-Gerber

e-mail: kk1@gcm.pl

A. Chmiel

e-mail: arturchmiel@wp.pl

M. Sosnowski

e-mail: maciej.sosnowski@gmail.com

Z. Gąsior

e-mail: zgasior@sum.edu.pl of the optimal prosthesis to be implanted in patients surgically treated for degenerative AS. 20 patients (F/M: 3/17; age: $69 \pm 6.5$ years) with severe degenerative AS were enrolled into the study. TTE, TEE and MSCT including AV calcium score (AVCS) assessment were performed in all patients. The values of AAd obtained in the long AV complex axis (TTE, TEE, MSCT) and in multiplanar perpendicular imaging (MSCT) were compared to the size of implanted prosthesis. The mean AAd was $24 \pm$

\author{
M. Trusz-Gluza \\ e-mail: trusz@cathlab.katowice.pl \\ M. Tendera \\ e-mail: michal.tendera@gmail.com
}

M. Jasiński - M. Krejca · A. Bochenek · S. Woś Departments of Cardiosurgery, Medical University of Silesia, Ziołowa 45/47, 40-635 Katowice, Poland e-mail: marekjasinski@yahoo.pl

M. Krejca

e-mail: mkrejca@wo.pl

A. Bochenek

e-mail: gcm-kkch1sek@slam.katowice.pl

S. Woś

e-mail: kkch2@gcm.pl 
$3.6 \mathrm{~mm}$ using TTE, $26 \pm 4.2 \mathrm{~mm}$ using TEE, and $26.9 \pm 3.2$ in MSCT ( $P=0.04$ vs. TTE). The mean diameter of the left ventricle out-flow tract in TTE $(19.9 \pm 2.7 \mathrm{~mm})$ and TEE $(19.5 \pm 2.7 \mathrm{~mm})$ were smaller than in MSCT $(24.9 \pm 3.3 \mathrm{~mm}, P<0.001$ for both). The mean size of implanted prosthesis $(22.2 \pm 2.3 \mathrm{~mm})$ was significantly smaller than the mean AAd measured by TTE $(P=0.0039)$, TEE $(P=0.0004), \quad$ and MSCT $(P<0.0001)$. The implanted prosthesis size correlated significantly to the AAd: $\mathrm{r}=0.603, P=0.005$ for TTE, $\mathrm{r}=0.592$, $P=0.006$ for TEE, and $\mathrm{r}=0.791, P<0.001$ for MSCT. Obesity and extensive valve calcification (AV calcium score $\geq 3177 \mathrm{Ag}$.U.) were identified as potent factors that caused a deterioration of both TTE and MSCT performance. The accuracy of AAd measurements in TEE was only limited by AV calcification. In multivariate regression analysis the mean value of the minimum and maximum AAd obtained in MSCT-multiplanar perpendicular imaging was an independent factor $(\mathrm{r}=0.802, P<0.0001)$ predicting the size of implanted prosthesis. In patients with AS echocardiography remains the main diagnostics tool in clinical practice. MSCT as a 3-dimentional modality allows for accurate measurement of entire AV complex and facilitates optimal matching of prosthesis size.

Keywords Aortic stenosis - Aortic valve replacement $\cdot$ Multi-slice spiral computed tomography · Transthoracic echocardiography . Transesophageal echocardiography

\section{Introduction}

Degenerative aortic stenosis (AS) is the most frequent valvular heart disease. In developed countries the occurrence of AS is estimated at $2-7 \%$ in population $>65$ years of age. Association between severe AS, negative prognosis and high mortality is universally acknowledged [1].

Transthorasic echocardiography (TTE) remains a routine, easily accessible and widely accepted method of aortic valve (AV) assessment. However, since it faces a number of practical limitations, final AS diagnosis and qualification for AV replacement
(AVR) may in some cases necessitate additional techniques. Transesophageal echocardiography (TEE) is most commonly employed to this end.

Three-dimensional modalities, including multislice computed tomography (MSCT) can strongly add to TTE and TEE evaluation. MSCT allows accurate assessment of the valve anatomy, particularly the annulus shape and diameter, valve morphology (bi- vs. tricuspid valve) and calcifications distribution. All these data are of utmost importance for interventional treatment planning, both surgical and transcatheter. In a few recently published reports, high sensitivity and specificity of MSCT was shown in planimetric calculations of AV area (AVA) leading to a conclusion that this method is at least as accurate as TTE and TEE [2-7]. Messika-Zeitoun et al. [8] confirmed the role of these methods in measuring the aortic annulus (AA) in patients with severe AS referred for transcatheter AV implantation. Until now, no data on efficacy of TTE, TEE, and MSCT in optimal selection of the prosthesis size has yet been reported. Regardless of the fact that conventional surgical approach allows for intra-operative sizing of the prosthesis, an accurate pre-operative assessment is necessary. Strict determination of the aortic annulus diameter (AAd) and shape seems to be of the essence especially for patient selection, choosing the prosthesis as well as implantation techniques.

We aimed to prospectively compare the accuracy of TTE, TEE and MSCT measurements of the AV complex and to analyze the role of multi-modality AAd assessment in the selection of the optimal prosthesis implanted in patients surgically treated for degenerative AS.

\section{Materials and methods}

\section{Patient population}

54 consecutive patients with severe degenerative AS defined as an effective orifice area (EOA) $<1.0 \mathrm{~cm}^{2}$ were diagnosed at the Department of Cardiology, Medical University of Silesia in 2009. From this population, $20(37 \%)$ patients (17 males, mean age: $69 \pm 6.5$ years, body mass index-BMI: $28.3 \pm$ $4.0 \mathrm{~kg} / \mathrm{m}^{2}$, body surface area-BSA: $1.97 \pm 0.2 \mathrm{~m}^{2}$ ) who underwent $\mathrm{AVR}$ and preoperative non-invasive 
multi-approach AS assessment (TTE, TEE, MSCT) were prospectively enrolled into the study. The indications for AVR were in agreement with the ESC recommendations [1]; the intra-operative sizing of the implanted AV prostheses was done correctly, i.e. according to the real, individual size of the aortic valve-aortic root complex. In the rest of initially screened subjects complete multi-approach AS assessment was impossible due to poor ultrasound window for TTE $(n=21)$, contraindications to contrast agent administration $(n=8)$, arrhythmia $(n=5)$. The other exclusion criteria included: coexisting hemodynamically important other valve diseases (moderate/severe aortic regurgitation, moderate/severe mitral regurgitation), congestive heart failure, thoracic deformation, renal failure, thyroid disease.

The multi-approach assessment was performed before cardiac surgery and the results of different methods were compared to each other as well as to the size of implanted prosthesis.

\section{Clinical data}

Clinical characteristics of study patients involved: medical history, physical examination (arterial pressure, heart rate, BMI), concomitant disease, standard TTE evaluation, type of the surgical procedure (AVR vs. AVR + CABG), AAd measured intra-operatively after the decalcification of the valve, type (artificial valve vs. bioprosthesis) and size of the implanted prosthesis.

Diagnosis of hypertension was based on blood pressure (BP) levels (systolic $\mathrm{BP} \geq 140 \mathrm{~mm} \mathrm{Hg}$ or diastolic $\mathrm{BP} \geq 90 \mathrm{~mm} \mathrm{Hg}$ ) or previously documented diagnosis and current antihypertensive treatment. Coronary angiography was performed in all patients and coronary artery disease (CAD) presence was defined as a lumen diameter narrowing of $\geq 70 \%$ in at least 1 of the 3 major epicardial coronary arteries. Obesity was defined as BMI $>30 \mathrm{~kg} / \mathrm{m}^{2}$. The study was approved by the Local Ethics Committee. All subjects enrolled into the study gave written informed consent.

Non-invasive multi-approach assessment of aortic valve

\section{Two-dimentional echocardiography}

Echocardiography was performed by an experienced sonographer with a standard ultrasound system

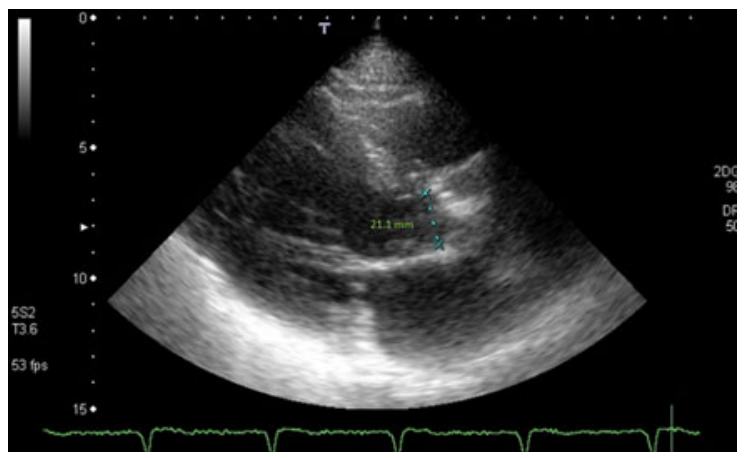

Fig. 1 a MSCT aortic annulus measurement-a long axis (LAX) perpendicular plane. b MSCT aortic annulus measurement-a long axis $(\mathrm{LAX})+90^{\circ}$ perpendicular plane

(Toshiba Aplio) equipped with a $3.5-1.75 \mathrm{MHz}$ transthoracic and a multiplane phased-array 2-7 MHz TEE transducers. Doppler echocardiographic indices of AS severity included maximal (Pmax) and mean (Pmean) transvalvular pressure gradients along with the effective orifice area (EOA) calculated from the continuity equation. Left ventricle end-diastolic volume (LV EDV), end-systolic volume (LV ESV), and ejection fraction (LV EF) were determined using modified Simpson's method.

All cardiac ultrasound examinations were performed in accordance with the American Society of Echocardiography and European Association of Echocardiography guidelines [9].

The following cross-section diameters of $\mathrm{AV}$ complex obtained during systole from a long axis in TTE (transthoracic parasternal long axis view) (Fig. 1) and TEE (135 ${ }^{\circ}$ mid-esophageal view) (Fig. 2) were analysed: LVOT (left ventricular outflow tract), AA, aortic bulb, STJ (sino-tubular junction), and ascending aorta. AVA planimetry was accomplished in a cross-sectional plane at the level where the valvular orifice was the smallest at the time of maximum valve opening.

\section{MSCT-scanning}

MSCT study was performed with Toshiba Aquilion 64 scanner (Toshiba, Japan). The detector collimation was $64 \times 0.5 \mathrm{~mm}$ with the rotation time of $0.4 \mathrm{~s}$. The tube current ranged from 330 to $430 \mathrm{~mA}$, the tube voltage was set at $120 \mathrm{kV}$ and pitch was 0.3 . Using a power injector system (Stellant, Medrad) a volume of $100-120 \mathrm{ml}$ of nonionic contrast agent with $400 \mathrm{mg} / \mathrm{ml}$ 


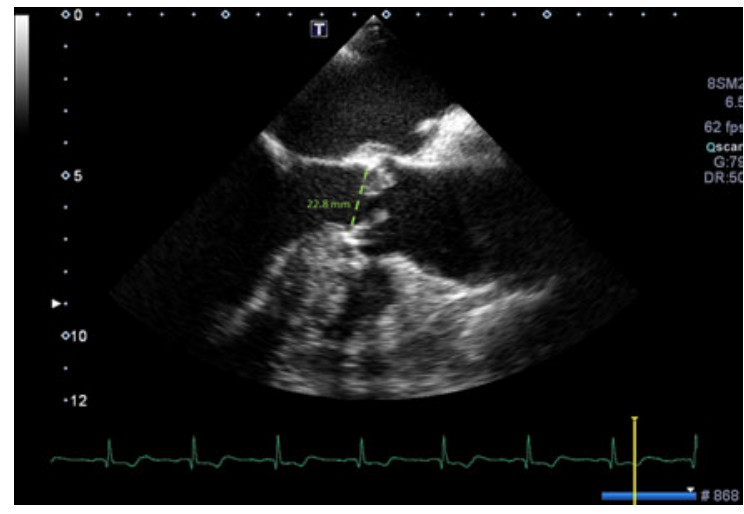

Fig. 2 Aortic annulus measurement in TTE-a parasternal long axis view

iodine content (Iomeprol, Iomeron 400, Bracco Int.), followed by saline chaser bolus of $30 \mathrm{ml}$, was injected in the antecubital vein at a mean flow rate of $5 \mathrm{ml} / \mathrm{s}$. Both volume and flow rate were adjusted to the patients body habitus. The ECG-gated scanning of the heart preceded by real time bolus tracking technique (SureStart, Toshiba Medical Systems) was performed. The scanning was triggered once the descending aorta opacity reached 180 H.U. Cardiac images ranging from the aortic arch to the apex were acquired during a single breathhold. Reconstruction image width was $0.5 \mathrm{~mm}$ with reconstruction interval of 0.3. Usage of multi-segmental reconstruction algorithms resulted in temporal resolution reaching approximately $150 \mathrm{~ms}$. Multiphase data set was reconstructed consisting of 10 different systolic cardiac phases in steps of $10 \%$ from 0 to $90 \%$ of the RR-interval.

The acquired images were transferred to a remote workstation (Vitrea2; Vital Images Inc., USA) for post-processing and evaluation (W/L 1000/200). The cardiac phase showing the most reliable data and that used for measurements of AV complex was seen mostly at $20 \%$ of the RR-interval. Depending on the scanning span, the dose length product (DLP) and the effective dose reached $1,200-1,400 \mathrm{mGy} / \mathrm{cm}^{2}$ and 19-22 mSv, respectively.

\section{MSCT_image analysis}

The measurements in MSCT were performed by an experienced radiologist using two methods. First, the AV complex was visualized in two perpendicular planes-LAX and LAX + 90 (Fig. 3a and b, respectively), the first of which reflected the transthoracic parasternal long axis view (Fig. 1) and $135^{\circ}$ midesophageal view (Fig. 2). The MSCT LAX plane was manually optimized with regards to anatomical details in each case by an investigator skilled in performing TTE and TEE exams. This approach allowed for a head-to-head comparison of the accuracy of different modalities. Fundamentally, a view was obtained where the continuity between the LVOT walls (i.e. aortic-mitral curtain, membranous ventricular septum or LV wall, depending on plane selection) and aortic sinuses was clearly visible. The annulus was then measured as the distance between the aortic cusps hinge points. If protruding calcifications were present at these particular points, they were included in the annulus dimension but only to the level of the above-mentioned LVOT-sinuses continuity line.

Secondly, to fully exploit the MSCT-related multiplanar imaging capability, we adjusted both perpendicular planes to achieve the measurements of minimum and maximum AAd. Thus, a more in-depth
Fig. 3 Aortic annulus measurement in TEE-a $135^{\circ}$ mid-esophageal view
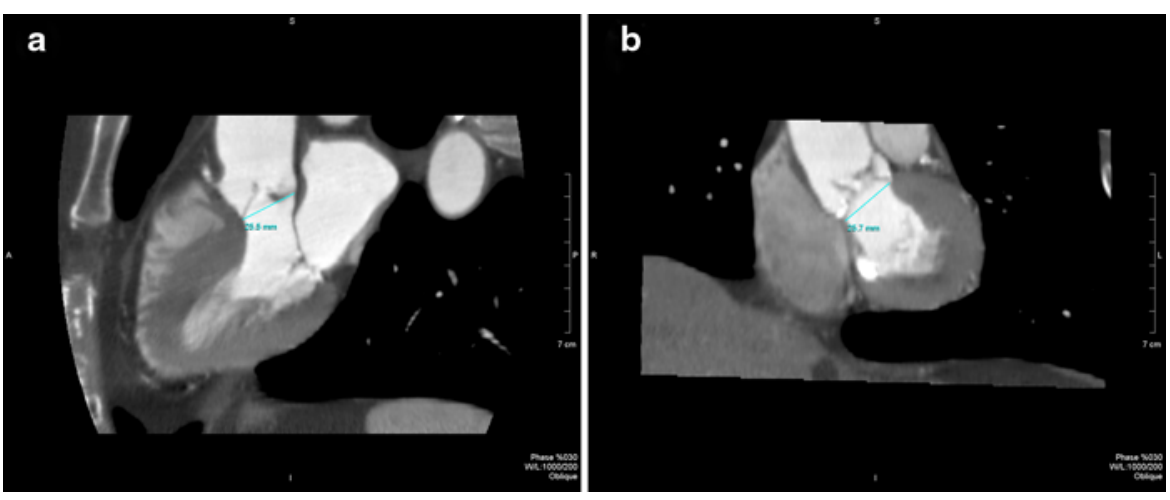
understanding of the elliptical character of aortic annulus became available. As a result, for each patient we calculated the mean values of AAd in LAX and LAX +90 planes along with the mean values of AAd in the perpendicular planes demonstrating minimum and maximum dimensions.

MSCT AVA planimetry was accomplished at the point when there was maximum valve opening with a cross-sectional plane positioned at the level of smallest valvular orifice.

\section{MSCT_calcium score}

Prior to contrast administration coronary artery calcium score (CACS) along with AV calcium score (AVCS) were also determined using a standardized MSCT imaging protocol with retrospective ECG gating. The detector collimation was $32 \times 0.5 \mathrm{~mm}$. The tube current ranged from 170 to $300 \mathrm{~mA}$, the tube voltage was set at $120 \mathrm{kV}$ and pitch was 0.4 . Axial images were reconstructed at $60 \%$ of the RR-interval, to achieve least motion artifacts, with an effective slice thickness of $3 \mathrm{~mm}$. Foci of calcium were identified by detection of at least three contiguous pixels (voxel size $=1.03 \mathrm{~mm}^{3}$ ) of peak density $\geq 130$ Hounsfield units (HU) within a coronary artery. The lesion-specific scores were computed as the product of the area of each calcified focus and peak CT number (scored as 1 if 131-199 HU, 2 if 200-299 HU, 3 if 300-399 HU, and 4 if $400 \mathrm{HU}$ or greater) according to the Agatston method [10]. AVCS was gauged cumulatively for valvular annulus and cusps.

\section{Statistical analysis}

All values are expressed as means \pm standard deviations or numbers and percentage. Continuous variables had a normal distribution that was validated by Fisher's test. One-way analysis of variance was used to compare the aortic complex diameters measured by TTE, TEE and MSCT. The intra-observer variability of AAd measurements was analyzed as the percentage difference between the two measurements: in TTE/TEE off-line assessment of the same image was performed; in MSCT the variability analysis required new reconstructions. Paired samples $t$ test was used to asses if systematic difference between the size of implanted prosthesis and the AAd in TTE, TEE, MSCT was present. Spearman's rank correlation coefficient analysis was used to evaluate the strength of the relationship between the variables. Regression equation was used to predict the size of implanted prosthesis from the aortic annulus diameter measured in MSCT. To asses the influence of factors: BMI, BSA, AVCS, age, gender, AAd in TTE, TEE, MSCT on the prediction of the size of implanted prosthesis a multiple stepwise linear regression was used. A value $P<0.05$ was considered statistically significant.

\section{Results}

Clinical data

All patients were in NYHA II/III and CCS I-III functional class.

The Doppler ultrasound indices of AS severity were as follows: Pmax: $75.8 \pm 21 \mathrm{mmHg}$, Pmean: $44.6 \pm$ 11.5, EOA: $0.7 \pm 0.2 \mathrm{~cm}^{2}$. The mean LV EDV was $136 \pm 64 \mathrm{ml}$, LV ESV: $60 \pm 36 \mathrm{ml}$, LV EF: $57.0 \pm$ $10.0 \%$. Mild aortic regurgitation was observed in 9 subjects.

Mean value of AVCS was 4,351 $\pm 2,782$ Agatston units (Ag.U.).

Systemic hypertension was diagnosed in $12(60 \%)$, CAD in $14(70 \%)$, obesity in $7(35 \%)$ subjects. Mean blood pressure was $136.8 \pm 10.4 \mathrm{mmHg}$, mean heart rate: $77.9 \pm 12.6 \mathrm{bpm}$ at the time of the TTE.

All patients underwent successful supra-annular aortic valve prosthesis implantation, in 10 (50\%) subjects accompanied by CABG. The mean value of AAd measured intra-operatively after the decalcification of the valve was $23.3 \pm 2.5 \mathrm{~mm}$. The following aortic valve prostheses were implanted: mechanical in $10(50 \%)$ patients (Bicarbon TR), bioprosthesis in 10 (50\%) patients (stented: Hancock IITR, Mosaic TR, and stentless: Freestyle TR).

\section{Intra-observer variability of TTE/TEE/MSCT}

The intra-observer coefficient of variation was $5.2 \%$ for TTE, $3.2 \%$ for TEE, and $3.1 \%$ for MSCT.

\section{TTE versus TEE versus MSCT}

The comparison of aortic complex diameters measured by TTE, TEE and MSCT is presented in Table 1. 
Table 1 Comparison of aortic complex diameters measured by TTE, TEE and MSCT

\begin{tabular}{lcccl}
\hline Diameter & TTE Mean \pm SD & TEE Mean \pm SD & MSCT Mean \pm SD & $P$ \\
\hline LVOT $(\mathrm{mm})$ & $19.9 \pm 2.7^{*}$ & $19.5 \pm 2.7^{*}$ & $24.9 \pm 3.3$ & $<0.001$ (vs. MSCT) \\
AAd $(\mathrm{mm})$ & $24 \pm 3.6^{*}$ & $26 \pm 4.2$ & $26.9 \pm 3.2$ & 0.04 (vs. MSCT) \\
Bulb $(\mathrm{mm})$ & $37 \pm 5.1$ & $35 \pm 4.4$ & $38 \pm 5.3$ & NS \\
STJ $(\mathrm{mm})$ & $31 \pm 4.6$ & $30 \pm 5.9$ & $31 \pm 4.7$ & NS \\
AoAsc $(\mathrm{mm})$ & $36 \pm 5.9$ & $35 \pm 7.7$ & $37 \pm 8.2$ & NS \\
AVA $\left(\mathrm{cm}^{2}\right)$ & $0.9 \pm 0.33$ & $0.86 \pm 0.30$ & $1.13 \pm 0.38$ & NS \\
\hline
\end{tabular}

LVOT left ventricle outflow tract, $A A d$ aortic annulus diameter, STJ sino-tubular junction, AoAsc ascending aorta, $A V A$ aortic valve area, TTE transthoracic echocardiography, TEE transoesophageal echocardiography, MSCT multi-slice computed tomography

*Significant correlation vs. MSCT

AAd versus size of AV prosthesis

The mean size of implanted prosthesis $(22.2 \pm 2.3)$ was significantly smaller than the mean value of AAd measured by TTE $(P=0.0039)$, TTE $(P=0.0004)$, and MSCT $(P<0.0001)$. The implanted prosthesis size correlated significantly to the AAd: $\mathrm{r}=0.603$, $P=0.005$ for TTE; $\mathrm{r}=0.592, P=0.006$ for TEE; and $\mathrm{r}=0.791, P<0.001$ for MSCT (Fig. $4 \mathrm{a}-\mathrm{c}$ ).

The accuracy of the methods used in prosthesis size selection was analyzed in relation to BMI and AVCS. Significant correlations between the size of the implanted prosthesis and the AAd in TTE and MSCT were found in non-obese patients $(P=0.015$,
$P<0.001$, respectively) and in patients with AVCS below the median value ( $<3,177$ Ag.U., $P=0.009$, $P=0.002$, respectively). The size of the implanted prosthesis correlated to the AAd in TEE both in nonobese patients and obese patients $(P=0.001$, $P=0.005$, respectively) as well as in patients with AVCS below the median value $(P=0.005)$ (Table 2).

The ability of non-invasive methods to predict the required prosthesis size based on AAd was found to be independent of the prosthesis type: artificial valve (TTE: $P=0.032$, TEE: $P=0.014$, MSCT: $P=$ 0.016 ) or bioprosthesis (TTE: $P=0.048$, TEE: $P=$ 0.038, MSCT: $P=0.003)$.
Fig. 4 Correlations between the size of the implanted prosthesis and AAd obtained in: a TTE: $r=0.603$, $\mathrm{y}=12.087+0.416 \mathrm{x}$, $P=0.005 ; \mathbf{b}$ TTE; $\mathrm{r}=0.592$, $\mathrm{y}=13.400+0.346 \mathrm{x}$, $P=0.00 ;$ c MSCT $\mathrm{r}=0.791$, $\mathrm{y}=8.571+0.467 \mathrm{x}$, $P<0.001$ on the LAX plane
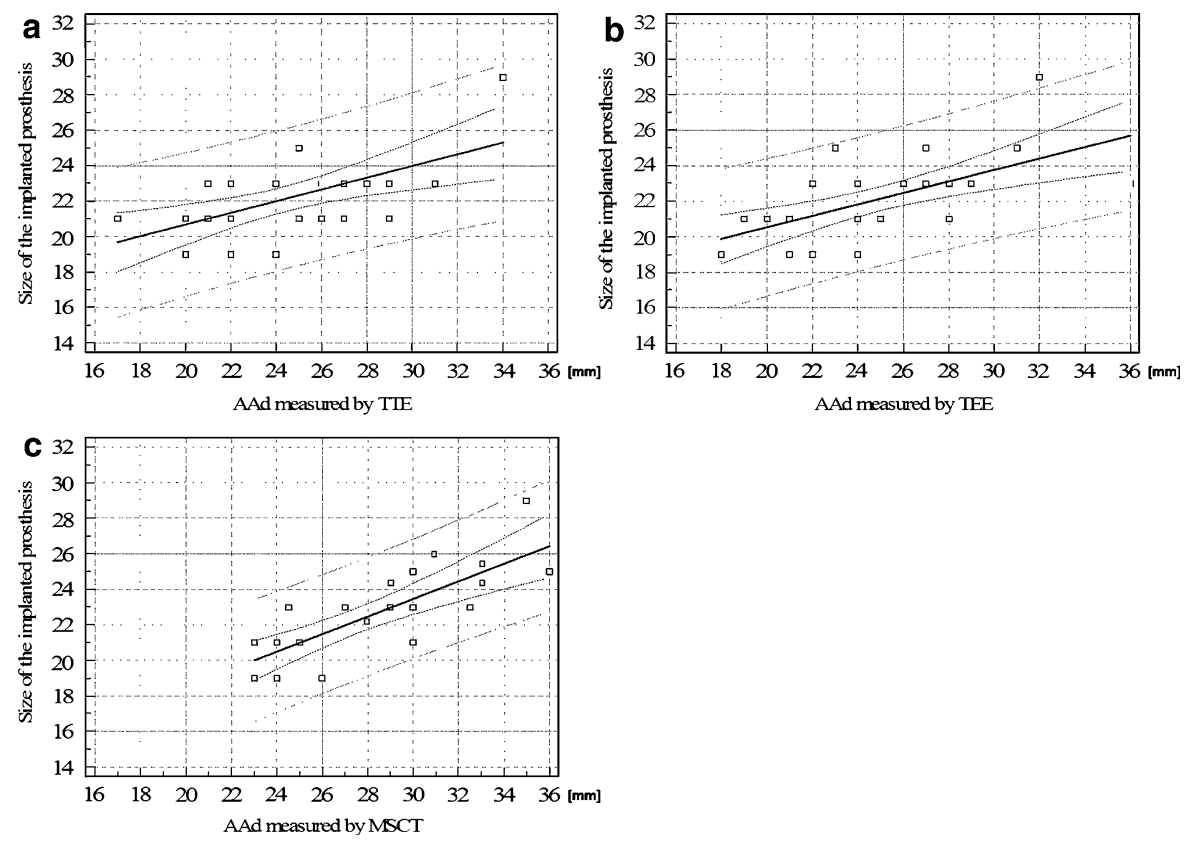
Table 2 Regression analysis between prosthesis size and TTE/TEE/MSCT-measured AAd diameter in relation to obesity and AVCS (non-obese patients $\mathrm{N}=13$, obese patients $\mathrm{N}=7$, patients with AVCS $<3,177$ Ag.U. $\mathrm{N}=10$, patients with $\mathrm{AVCS} \geq 3,177$ Ag.U N = 10)

\begin{tabular}{|c|c|c|}
\hline & $\mathrm{r}$ & $P$ \\
\hline \multicolumn{3}{|l|}{ TTE } \\
\hline \multicolumn{3}{|l|}{ Obesity } \\
\hline Non-obese & 0.563 & 0.015 \\
\hline Obese & 0.474 & 0.140 \\
\hline \multicolumn{3}{|l|}{$A V C S$} \\
\hline AVCS < 3,177 Ag.U. & 0.805 & 0.009 \\
\hline AVCS $\geq 3,177$ Ag.U. & 0.597 & 0.052 \\
\hline \multicolumn{3}{|l|}{ TEE } \\
\hline \multicolumn{3}{|l|}{ Obesity } \\
\hline Non-obese & 0.834 & 0.001 \\
\hline Obese & 0.872 & 0.005 \\
\hline \multicolumn{3}{|l|}{$A V C S$} \\
\hline AVCS < 3,177 Ag.U. & 0.635 & 0.005 \\
\hline AVCS $\geq 3,177$ Ag.U. & 0.534 & 0.091 \\
\hline \multicolumn{3}{|l|}{ MSCT } \\
\hline \multicolumn{3}{|l|}{ Obesity } \\
\hline Non-obese & 0.907 & $<0.001$ \\
\hline Obese & 0.308 & 0.196 \\
\hline \multicolumn{3}{|l|}{$A V C S$} \\
\hline AVCS < 3,177 Ag.U. & 0.884 & 0.002 \\
\hline AVCS $\geq 3,177$ Ag.U. & 0.508 & 0.072 \\
\hline
\end{tabular}

In multivariate regression analysis the AAd measured in MSCT was an independent factor $(r=0.791$, $P<0.0001)$ predicting the size of implanted prosthesis.

MSCT-related multiplanar imaging and AAd estimation

MSCT-related multiplanar imaging revealed nonsignificant differences between the mean values of AAd measured in the following planes: LAX and LAX + 90; and as minimum and maximum AAd in the perpendicular planes of measurement (Table 3). However, the comparison of the minimum and maximum AAd in consecutive individuals showed difference up to $5.1 \mathrm{~mm}$. The implanted prosthesis size correlated significantly to the AAd in all planes
Table 3 Regression analysis between prosthesis size and aortic annulus diameter measured in MSCT-related multiplanar imaging

\begin{tabular}{llll}
\hline & $\begin{array}{l}\text { AAd }(\mathrm{mm}) \\
\text { Mean }+\mathrm{SD} \\
\text { (range) }\end{array}$ & $\mathrm{r}$ & $P$ \\
& $26.9 \pm 3.2$ & 0.677 & 0.001 \\
& $(21.9-32.6)$ & & \\
1. LAX & $27.6 \pm 3.6$ & 0.614 & 0.004 \\
2. LAX $+90^{\circ}$ & $(21.0-33.2)$ & & \\
Mean value: 1 and 2 & $27.3 \pm 3.5$ & 0.655 & 0.002 \\
3. Minimum AAd & $26.1 \pm 3.1$ & 0.688 & 0.001 \\
& $(21.0-34.0)$ & & \\
4. Maximum AAd & $28.8 \pm 3.6$ & 0.675 & 0.001 \\
& $(21.8-34.4)$ & & \\
Mean value: 3 and 4 & $27.4 \pm 3.5$ & 0.699 & 0.001 \\
\hline LAX long axis, AAd aortic annulus diameter & &
\end{tabular}

of measurement (Table 3). In multivariate regression analysis the mean value of the minimum and maximum AAd optimally fitted the model $(\mathrm{r}=$ $0.802, P<0.0001)$.

\section{Discussion}

Current standard pre-procedural imaging in degenerative AS is based on 2D echocardiography, however, the role of other imaging modalities, including MSCT, is emerging. In the present study we compared 3 methods of AVA and AV complex measurements, namely TTE, TEE, and MSCT in patients surgically treated for degenerative AS. Additionally, we analyzed the role of multi-modality AAd assessment in selection of the optimal prosthesis to be implanted.

We found some differences between the AV complex dimensions measured by TTE, TEE and MSCT. The LVOT values in TTE and TEE were smaller than in MSCT. A similar trend was observed regarding to the AAd-smaller diameters of AAd were obtained in TTE as compared to MSCT. Probably the severe calcifications and the 3-dimentional structure of LVOT and AAd may explain these discrepancies. On the other hand, the methods used in the study were in accord as to the measurements of the upper part of aortic root, aortic bulb, and STJ, as well as the diameter of ascending aorta. A lower 
degree of calcification in this region may justify a better visualization. There were also no differences in the evaluation of AVA.

According to the literature the MSCT-determined AVA strongly correlates with cardiac ultrasound findings and the modality shows excellent sensitivity and specificity to detect severe stenosis [2-6, 10-13]. Messika-Zeitoun et al. [8] presented results comparable to our findings. They found that in patients referred for transcatheter aortic valve implantation the highest AAd values were obtained by MSCT as compared to 2D-echocardiography. The difference between the AAd estimated in MSCT and TEE or TEE was larger than that observed between TTE and TEE. In the study by Tops et al. [14], a similar trend toward lower AAd in TTE as compared to MSCT was registered. However, MSCT measurements were done in the sagittal view. AV planimetry by MSCT was feasible in all patients, while only in 78 or $57 \%$ of patients by TEE or TTE, respectively.

Since the AAd is of key significance in prosthesis selection, the TTE, TEE and MSCT AAd measurement results were compared to the prosthesis size. Regardless of modality, non-invasively determined AAd values were higher than the size of subsequently implanted prosthesis-the non-invasive methods overestimated the size of the annulus; however, at the same time, the AAd values correlated well with the size of the implanted prosthesis. This remains in keeping with well-known data showing a need for systematic difference to be taken into account while using TTE, TEE and MSCT for therapeutic decision making [8, 14]. The most significant correlations between AAd dimension and the prosthesis size were achieved for MSCT. It should be pointed out that these relations were consistent for both AAd measurement in long axis and mean AAd calculated from two other perpendicular planes. In the multivariate analysis the AAd measured by MSCT was the only independent factor significantly influencing the prosthesis size. Moreover, the mean value of minimum and maximum AAd optimally predicted the size of prosthesis. It indicates that MSCT, allows for accurate estimation of the prosthesis size. This accurate preoperative valve size estimation may help to avoid postoperative patient-prosthesis mismatch (PPM) by scheduling those with high risk of PPM (predicted small prosthesis size) to special procedures namely stentless implantation or aortic root enlargement.
This can allow for the optimal selection of implantation techniques, namely supraannular versus intraannular and stentless (homograft, xenograft) versus stented or mechanical prostheses [15]. On the other hand, in standard surgical procedure intra-operative sizing is performed. However, this method has its own limitations regarding the non-physiological hemodynamic conditions associated with cardiopulmonary bypass. The fact that intra-operative sizing is performed during diastole makes difficult to compare the results to the TTE and TEE evaluation that is routinely measured in systole. This is why we finally compared pre-operative AAd assessment to the size of the implanted prosthesis not to the result of intraoperative sizing.

Detailed 3D analysis, feasible mainly by means of MSCT [1, 16], but also in 3D echocardiography [17], demonstrates that AA is not necessarily circular but often elliptical. Consequently, MSCT imaging promotes better understanding of $\mathrm{AV}$ complex anatomy simultaneously allowing more precise estimation of mean AAd. In our study MSCT-related multiplanar imaging revealed non-significant differences between the mean values of AAd measured in the perpendicular planes. However, a comparison of the minimum and maximum AAd in consecutive individuals showed differences up to $5.1 \mathrm{~mm}$. Thus, the complex assessment of AAd in MSCT allowed the eccentricity of the AV orifice in some patients to be revealed, which is important from the practical point of view. As was mentioned above, the mean value of the minimum and maximum AAd optimally predicted the size of prosthesis. The problem of AAd eccentricity in patients with AS referred to transcatheter aortic valve replacement was presented in the study by Messika-Zeitoun et al. [8]. Authors measured long- and short axis diameters of the AA at the level of the virtual basal ring and found differences up to $5.8 \mathrm{~mm}$ - similar to these observed in our study. The problem of AA eccentricity would probably be even more important for patients with a degenerated bicuspid aortic valve.

Despite the low number of patients, the study elicited significant factors limiting the accuracy of the multimodality approach in AAd estimation. Obesity was identified as potent factor deteriorating both TTE and MSCT performance. This potential limitation might be significantly reduced in MSCT by applying a different X-ray tube settings adjusted to the 
patient's body size. AV calcification, typical for degenerative AS, hampered the accuracy of all of the methods investigated. Measurements remained feasible even in the case of massive calcification but at a cost of lower precision. We are inclined to conclude that high AVCS should raise greater alertness at interpreting AAd estimation. It is well-documented in the literature that the presence of massive calcifications of the annulus and cusps may render TTE and TEE (especially the 2D examination) and also render magnetic resonance unreliable, including the evaluation of the geometry of the annulus $[2,18,19]$. On the other hand, MSCT allows to show the exact location of calcific deposits. The detailed knowledge about aortic root and valve calcifications is important for better understanding the pathology complicating surgical and percutaneous AVR [20].

The main limitation of the study was relatively small number of enrolled patients.

$35 \%$ of patients were obese $\left(\mathrm{BMI}>30 \mathrm{~kg} / \mathrm{m}^{2}\right)$ which probably affected the process of measurements taking. To avoid Doppler-flow-parameters-based AVA miscalculation caused by impaired hemodynamics (e.g. LV dysfunction, severe aortic regurgitation) we only examined ,pure” AS patients with preserved LVEF. In the study only patients for whom a complex multi-approach evaluation was feasible were enrolled into the final analysis and $63 \%$ of patients were excluded mainly due to the poor acoustic window for TTE. Taking this into account, TEE and MSCT seem even more comprehensive.

64-MSCT allows most detailed reconstruction of the AV complex. However, the exposure to radiation and administration of iodine-based contrast agent must be considered in adjusting MSCT protocol for individual patients. ECG-controlled tube current modulation (prospective ECG-gating or maximal ECG-pulsing), typically applied to reduce radiation exposure [21], was not recommended in our protocol since we needed full cardiac cycle to accurately assess coronaries during diastole and take AV complex measurements at peak systole. The application of dose saving algorithms in our protocol might critically compromise image quality, especially during systole. MSCT acquisition without contrast administration might potentially enable overall assessment of root size, but does not permit annulus description [2]. Degenerative AS occurs in older patients and available data suggests that MSCT-related radiation dose may not significantly increase the life-time risk of cancer in this age group [14, 22]. Nevertheless, the MSCT benefit should be considered individually.

Summarizing, in patients with AS echocardiography remains the main diagnostics tool in clinical practice. AV MSCT planimetry along with entire AV complex measurement is accurate and allows optimal estimation of the required prosthesis size, what may influence type of surgical treatment chosen. Because of exposure to radiation, MSCT has not become a method of choice in AS assessment yet. However, MSCT possibly will become an alternative to standard echocardiography especially in patients scheduled for AVR with poor acoustic window and narrow aortic root. New 3-dimensional modalities seem to be promising tools probably due to the complex AV structure and elliptical shape of the aortic annulus. Severe AV calcification continues to limit the accuracy of all of the methods.

Open Access This article is distributed under the terms of the Creative Commons Attribution Noncommercial License which permits any noncommercial use, distribution, and reproduction in any medium, provided the original author(s) and source are credited.

\section{References}

1. Vahanian A, Baumgartner H, Bax J et al (2007) Task force on the management of valvular hearth disease of the European society of cardiology; ESC committee for practice guidelines. ESC Guidelines on the management of valvular heart disease. Eur Heart J 28:230-268

2. Schoenhagen P, Tuzcu ME, Samir R et al (2009) Threedimensional imaging of the aortic valve and aortic root with computed tomography: new standards in an era of transcatheter valve repair/implantation. Eur Heart J 30: 2079-2086

3. Feuchtner GM, Dichtl W, Friedrich GJ et al (2006) Multisliced computed tomography for detection of patients with aortic valve stenosis and quantification of severity. J Am Coll Cardiol 47:1410-1417

4. Alkadhi H, Wildermiuth S, Plass A et al (2006) Aortic stenosis: comparative evaluation of 16-detector CT and echocardiography. Radiology 240:47-55

5. Feuchtner GM, Muller S, Grander W et al (2006) Aortic valve calcification as quantified with multislice computed tomography predicts short-term clinical outcome in patients with asymptomatic aortic stenosis. J Heart Valve Dis 15:494-498

6. Feuchtner GM, Muller S, Bonatti J et al (2007) Sixty-four slice CT evaluation of aortic stenosis using planimetry of the aortic valve area. Am J Roentgenol 189:197-203 
7. Shah RG, Novaro GM, Blandon RJ et al (2009) Aortic valve area: meta-analysis of diagnostic performance of multi-detector computed tomography for aortic valve area measurements as compared to transthoracic echocardiography. Int J Cardiovasc Imaging 25(6):601-609

8. Messika-Zeitoun D, Serfaty JM, Brochet E et al (2010) Multimodal assessment of the aortic annulus diameter: implications for transcatheter aortic valve implantation. J Am Coll Cardiol 55:186-194

9. Baumgartner H, Hung J, Bermejo J et al. (2009) Echocardiographic assessment of valve stenosis: EAE/ASE recommendations for clinical practice. Am Soc Echocardiogr 22(1):1-23; Erratum in: J Am Soc Echocardiogr 22(5):442

10. Agatston AS, Janowitz WR, Hildner FJ et al (1990) Quantification of coronary artery calcium using ultrafast computed tomography. J Am Coll Cardiol 15:827-832

11. La Bounty TM, Sundaram B, Agarwal P et al (2008) Aortic valve area on 64-MDCT correlates with transesophageal echocardiography in aortic stenosis. Am J Roentgenol 191 (6):1652-1658

12. Lembcke A, Kivelitz DE, Borges AC et al (2009) Quantification of aortic valve stenosis: head-to-head comparison of 64-slice spiral computed tomography with transesophageal and transthoracic echocardiography and cardiac catheterisation. Invest Radiol 44(1):7-14

13. Lembcke A, Woinke M, Borges AC et al (2009) Grading of aortic valve stenosis at 64-slice spiral computed tomography: comparison with transthoracic echocardiography and calibration against cardiac catheterization. Invest Radiol 44(6):360-368

14. Tops LF, Wood DA, Delgado V et al (2008) Noninvasive evaluation of the aortic root with multislice computed tomography implications for transcatheter aortic valve replacement. JACC Cardiovasc Imaging 1(3):321-330
15. Jasinski M, Hayton J, Kadziola Z et al (2002) Hemodynamical performance after stented vs stentless aortic valve replacement. J Cardiovasc Surg 43(3):313-317

16. Doddamani S, Grushko MJ, Makaryus AN et al (2009) Demonstration of left ventricular outflow tract eccentricity by 64-slice multi-detector CT. Int J Cardiovasc Imaging 25(2):175-181

17. Doddamani S, Bello R, Friedman MA et al (2007) Demonstration of left ventricular outflow tract eccentricity by real time 3D echocardiography: implications for the determination of aortic valve area. Echocardiography 24 (8):860-866

18. Schultz C, Weustink A, Piazza N et al (2009) Geometry of degree of apposition of the corevalve revalving system with multislice computed tomography after implantation in patients with aortic stenosis. J Am Coll Cardiol 54 (10):911-918

19. Westermann Y, Geigenmuller A, Elgeti T et al. (2009) Planimetry of the aortic valve orifice area: comparison of multislice spiral computed tomography and magnetic resonance imaging. Eur J Radiol 22. [Epub ahead of print]

20. Rivard AL, Bartel T, Bianco RW et al (2009) Evaluation of aortic root and valve calcifications by multi-detector computed tomography. J Heart Valve Dis 18(6):662-670

21. Jakobs TF, Becker CR, Ohnesorge B et al (2002) Multislice helical CT of the heart with retrospective ECG gating: reduction of radiation exposure by ECG-controlled tube current modulation. Eur Radiol 12:1081-1086

22. Brenner DJ (2004) Radiation risks potentially associated with low-dose CT screening of adult smokers for lung cancer. Radiology 231:440-445 